\begin{tabular}{|l|l} 
REVISTA & $\begin{array}{l}\text { Revista Educación } \\
\text { ISSN: 0379-7082 } \\
\text { ISSN: } 2215-2644 \\
\text { revedu@ gmail.com } \\
\text { Universidad de Costa Rica } \\
\text { Costa Rica }\end{array}$
\end{tabular}

\title{
Evaluación de competencias en educación superior: conceptos, principios y agentes
}

\author{
Bonnefoy Valdés, Nicolás \\ Evaluación de competencias en educación superior: conceptos, principios y agentes \\ Revista Educación, vol. 45, núm. 2, 2021 \\ Universidad de Costa Rica, Costa Rica \\ Disponible en: https://www.redalyc.org/articulo.oa?id=44066178032 \\ DOI: https://doi.org/10.15517/revedu.v45i1.43444
}

\section{(c) $(1) \Theta(9$}

Esta obra está bajo una Licencia Creative Commons Atribución-NoComercial-SinDerivar 3.0 Internacional. 
Revisiones bibliográficas

\section{Evaluación de competencias en educación superior: conceptos, principios y agentes}

Higher Education Skills Assessment: Concepts, Principles and Stakeholders

Nicolás Bonnefoy Valdés

Pontificia Universidad Católica de Valparaíso, Chile

nicobonneva@gmail.com

DOI: https://doi.org/10.15517/revedu.v45i1.43444 Redalyc: https://www.redalyc.org/articulo.oa?

(iD https://orcid.org/0000-0002-4805-859X $\mathrm{id}=44066178032$

Recepción: 29 Septiembre 2020 Aprobación: 25 Noviembre 2020

\section{Resumen:}

Las instituciones de educación superior actualmente están adoptando, a nivel mundial, una nueva forma de orientar la enseñanza de los contenidos a las competencias. Por su carácter de situado y complejo, la competencia requiere de otros modos de evaluación. El propósito de este artículo es presentar un análisis de cómo la evaluación de las competencias en el aula se formula, diseña e implementa mediante la revisión de literatura especializada como metodología selecta. El enfoque de este artículo fue de la evaluación de competencias a nivel aula, desde la perspectiva del personal docente. Los resultados del artículo permitieron distinguir y analizar los conceptos asociados a la evaluación de competencias, los principios por los que una competencia debe ser evaluada; los estándares, evidencias y otras consideraciones, y los ejemplos de pasos para realizarla. Es relevante profundizar en los y las agentes involucradas: estudiantes, docentes e instituciones. Se concluye que la evaluación de competencias tiene un cuerpo teórico extenso, y que para su adopción es vital el diálogo entre agentes.

Palabras Clave: Competencias, Evaluación, Educación universitaria, Evaluación del estudiantado.

\section{ABstract:}

Higher education institutions worldwide are currently adopting a new way to steer learning from content to competency-based. Given its complex nature, competency-based education must be assessed differently. This article examines how to formulate, design and implement competency-based assessments in the classroom, using a review of pertinent literature as the selected methodology. This article focuses on competency-based assessment in the classroom from the perspective of the instructor. Results help distinguish and analyze concepts associated with competency-based assessments, the underlying principles, standards, evidence and other considerations, as well as examples for its applications and the stakeholders involved: namely students, teachers and institutions. The article concludes that competency-based assessments have an extensive theoretical makeup and that dialogue among its stakeholders is essential for its adoption.

KEYWORDS: Competences, Assessment, University Education, Students Assessment.

\section{INTRODUCCIÓN}

La educación se contempla como un derecho y como un instrumento imprescindible para el ejercicio de otros derechos también universales; entre ellos, el derecho al desarrollo (Murga-Menoyo, 2015). El sistema de formación superior necesita centrarse en proporcionar una base consistente donde enmarcar la capacidad de innovar y de adaptarse de forma rápida y eficaz, al actualizar constantemente las competencias requeridas en la vida laboral (Villa y Poblete, 2011). La respuesta que han dado las instituciones de educación superior en el mundo para consolidar el desarrollo de sus estudiantes está en la transición a la formación por competencias. A nivel de instituciones, proyectos como el Tuning Academy fomentan en las universidades de más de 120 países ${ }^{[1]}$ un sistema intercultural para desarrollar aprendizajes, centrado en el estudiantado y basado en competencias y resultados. Estas experiencias han trascendido sus orígenes europeos (Birtwistle, Brown y Wagenaar, 2016) y se han instalado en Estados Unidos (Nodine, 2016), América Latina (Beneitone y Yarosh, 2015), Asia y África (Isaacs, 2017). 
La formación de competencias requiere repensar la labor del personal docente universitario en varias facetas, donde una es el cómo estas serán evaluadas. Este artículo tiene como propósito servir de introducción a una discusión sintética y fundamentada para docentes, curriculistas o cualquier otra persona profesional involucrada en procesos de mejora e innovación curricular; esto al explorar los aportes que diversos autores y autoras han elaborado en los últimos años. Como objetivos de discusión se abordan tres ámbitos de la evaluación de competencias que hipotéticamente presentan problemas al intentar integrarlos a una institución: la diversidad de miradas y complejidad de definir y situar la evaluación de competencias en contraste con la evaluación del conocimiento, los principios, estándares y las rutas a seguir para a la integración de una evaluación por competencias, y las consideraciones que se deben hacer sobre los y las agentes involucradas en el acto de evaluar competencias.

En este artículo se presenta una síntesis de las variables a considerar para evaluar competencias, organizada según los objetivos de discusión a resolver: primero se consultan los conceptos de la evaluación de competencias para estudiar los constructos teóricos desde donde plantear y diseñar la evaluación; en segundo lugar, se exploran los estándares y pasos necesarios para facilitar una evaluación por competencias mediante el contraste de diferentes personas autoras; en tercer lugar, se exploran las y los agentes involucrados, sus roles y las nuevas exigencias que deben cumplir.

\section{Metodología}

Para la elaboración de este artículo se consultó en la literatura existente (artículos científicos indexados), con énfasis en publicaciones recientes en inglés y español; para ello se utilizaron las bases de artículos Google Académico y Eric. El enfoque de este artículo fue de la evaluación de competencias a nivel aula desde la perspectiva del personal docente. En esta área se encontró más conceptualización de competencias y su evaluación en la literatura hispana. En este artículo no se hablará de los enfoques donde el interés está en la necesidad de evaluar masiva y estandarizadamente (Cubero-Ibáñez, Ibarra-Sáiz y Rodríguez-Gómez, 2018; Zlatkin-Troitschanskaia, Shavelson y Kuhn, 2015), para favorecer los procesos de certificación de las competencias.

\section{Resultados}

A continuación, se presentan los resultados de la revisión bibliográfica. Se analizan las definiciones de competencia y los principios detrás del concepto de evaluación de competencias. Se abordan tópicos que son consecuencia de estos principios, la necesidad de estándares, evidencias y consideraciones, y los pasos que requiere. Se abordan los roles que toma el estudiantado, el personal docente y las instituciones en la evaluación de competencias.

\subsection{Concepto de competencia}

El Centro Europeo para el Desarrollo de la Formación Profesional define competencia como "Capacidad de una persona para aplicar correctamente los resultados de aprendizaje obtenidos a un contexto concreto (en la educación, el trabajo o el desarrollo personal o profesional)" (CEDEFOP, 2008, p. 48). Las competencias se enmarcan en un enfoque constructivista de la educación, ya que plantean la necesidad de lograr en el estudiantado la transferencia de saberes a contextos inmediatos y de largo plazo (Manríquez, 2012). Marcos como el Europeo son el producto de las propuestas originales e influentes de autores como Perrenoud (2004), que define a la competencia como la capacidad de movilizar varios recursos cognitivos para hacer frente a un tipo de situaciones; Tardif (2008), que la operacionaliza como un saber actuar complejo que se apoya sobre la 
movilización y la utilización eficaz de una variedad de recursos, o Le Boterf (2000), que la entiende como un saber combinatorio construido por el sujeto-aprendiz, que construye la competencia a partir de la secuencia de actividades de aprendizaje que movilizan múltiples conocimientos especializados.

Tejada (1999) define el concepto de competencias como "Un conjunto de conocimientos, procedimientos y actitudes combinados, coordinados e integrados en el ejercicio profesional, definibles en la acción, donde la experiencia se muestra como ineludible y el contexto es clave" (p. 27). Según esta definición, Tejada y Ruiz (2016) advierten que "La competencia no reside en los recursos (capacidades) sino en la movilización misma de los recursos" (p. 20). Las competencias están configuradas por componentes cognitivos, axiológicos y procedimentales, además de elementos del mundo subjetivo de la persona (Murga-Menoyo, 2015)

La síntesis sobre conceptualización de competencia profesional (Muñoz y Araya, 2017; Tejada y Ruiz, 2016) postula 4 principios:

1. No es una suma/adición, sino un ensamblaje con una dinámica de interacción entre elementos;

2. ha de tratarse la competencia más como un proceso que como una suma de recursos;

3. ser competente (dominio de la acción) es diferente de tener competencias (tener recursos);

4. no es suficiente poseer los recursos - saberes-, es necesario saberlos utilizar bien en contextos particulares.

Cano (2008) compila 18 definiciones diferentes de competencia encontradas en la literatura, e incluye su propia síntesis, con tres elementos fundamentales. Según su definición, las competencias: a) articulan conocimiento conceptual, procedimental y actitudinal, pero van más allá; b) se vinculan a rasgos de personalidad, pero se aprenden; c) toman sentido en la acción, pero con reflexión.

No existe una definición definitiva al hablar de competencias. Por esta razón, ya existe una confusión de base al no tener de antemano definido lo que se busca evaluar. Por ello, es necesario delimitar el conjunto de definiciones a algunas personas autoras centrales, y desde allí idear una evaluación de las competencias.

\subsubsection{Complejizar la evaluación}

Definir a la competencia como un conjunto de saberes complejos (Tardif, 2008; Tejada y Ruiz, 2013, 2016), corresponde que en su evaluación se debe realizar un análisis cuidadoso de los saberes implicados. La complejidad implica que la integración de la teoría a la práctica debe ser consistente y coherente (Jornet, González, Suárez y Peraless, 2011). La conceptualización de competencias realza la acción, la experiencia y el contexto socioprofesional, y sus medidas evaluativas deben reflejarlo. Tobón (2013) resalta que toda competencia tiene diferentes niveles de complejidad, y es necesario que se aborde esto para evitar agrupar al estudiantado en términos de competente/no competente.

Se espera que la educación superior potencie la relación entre lo académico y lo laboral, y que el estudiantado ofrezca respuestas complejas, holísticas e integrales a los problemas de la vida real (Moreno, 2012), más no es su objeto final focalizarse solo en este tipo de vinculaciones. Una evaluación que propague la mecanización de las competencias como una forma de reproducir el modelo productivo, estaría fallando en su concepción como modelo formativo (García-Sanz, 2014). La evaluación debe guiar a unir estrategias para promover la adquisición, mantenimiento y generalización de las competencias (Rodríguez, Rodríguez y Silva, 2017).

El planteamiento de Tejada y Ruiz $(2013,2016)$ conlleva tres dimensiones relevantes a considerar en la formación por competencias: a) el perfil profesional como referente del diseño curricular, b) el espacio formativo que integra la institución formativa y la institución sociolaboral y c) el tiempo formativo que se proyecta a lo largo de toda la vida, donde es insuficiente la formación inicial y da protagonismo a la formación continua. 
Una evaluación centrada en el estudiantado, como es en el caso de las competencias, significa que la persona estudiante requiere de retroalimentación inmediata, frecuente y formativa (Tejada y Ruiz, 2016; Villa y Poblete, 2011). Por esta complejidad, existe un vínculo en la literatura entre la formación por competencias con la evaluación formativa (Sadler,1989), entendida como conductora a lo largo de los ciclos de aprendizaje, y cuyo enfoque está en que el alumnado aprenda qué es lo que ha hecho bien y qué pasos debe dar para mejorar (González y Wagenaar, 2008). Muñoz y Araya (2017) sugieren visualizar el enfoque evaluativo por competencias en su sentido formativo y como una oportunidad de desarrollo de aprendizajes.

La evaluación formativa centra la atención en las actividades de aprendizaje y su mejora, la retroalimentación continua, la reflexión sobre el proceso, la autoevaluación y la colaboración (Tejada y Ruiz, 2013) mientras que la evaluación sumativa, ligada a la evaluación de las competencias, se conecta con el desempeño, niveles de logro, evidencias y gestión eficaz de situaciones.

Esta situación de evaluación formativa posibilita vislumbrar aspectos que deben ser reformulados para potenciar los nuevos requerimientos de las competencias. Parte de la complejidad de trabajar con competencias es que "la evaluación no solo debe medir el aprendizaje, sino también favorecerlo" (Tejada y Ruiz, 2016, p. 27). Esta lógica significa que la evaluación deja de estar emparejada con una sola función (la cualificación del estudiantado), y adquiere varias funciones; además, permite entender que la evaluación conlleva un cambio de paradigma:

\footnotetext{
Estas asunciones de cambio paradigmático evidente, conllevan automáticamente repensar el hecho evaluativo y su proceso. Es decir, desde el qué evaluar (objeto, indicadores, criterios,...) hasta cómo evaluar (modelos y estrategias), pasando por el para qué evaluar (finalidades y toma de decisiones), el cuándo evaluar o momento evaluativo y con qué evaluar, aludiendo a los instrumentos, técnicas o dispositivos de recogida de información y quién evaluará (agentes evaluadores) (Tejada y Ruiz, 2016, p. 28).
}

Otro concepto con el que se cruza la evaluación de competencias es la evaluación auténtica (Pérez, 2014), que se refiere al desempeño en situaciones y escenarios reales que permiten reconocer las competencias adecuadas para actuar conforme a lo que se espera. Por lo tanto, la evaluación de competencias como proceso complejo debe orientarse hacia la acción, como referente situaciones reales o simuladas de trabajo (Cordero, 2018; Moreno, 2012).

En la necesidad de aprendizaje comprehensivo y situado es que se da una intersección entre lo formativo y lo auténtico, cuestión que requiere la evaluación de competencias. Algunas consecuencias de este cambio de paradigma se explorarán en el siguiente apartado.

\subsection{Principios de la Evaluación de competencias}

Tejada y Ruiz $(2013,2016)$ están de acuerdo en afirmar que la evaluación de competencias tiene orígenes en una forma de aprendizaje que es experiencial y situado, con sus orígenes en los propios planteamientos de la metodología de la exploración de Dewey (1989), del concepto de andamiaje para la formación de profesores competentes de Bruner (1978), y la idea del profesor como mediador del conocimiento en Vygotsky (1988), ya que en la formación por competencias todo proceso de aprendizaje es una integración, tanto de información como de experiencia. Manríquez (2012) deriva la importancia de un modelo basado en competencias, en un enfoque en la acción que atribuye significado al conocimiento impartido. El aprendizaje sin atribución de significados personales es mecánico, no significativo.

La labor en la evaluación de competencias es transformar el principio del aprendizaje significativo en rediseños o formulaciones que hagan de las competencias un objeto a ser evaluado (Muñoz y Araya, 2017) y que responda a concebir la evaluación como un proceso democrático, participativo y liberador, que demanda la implicación consciente del alumno como sujeto que aprende (Kemmis, 1988). El aprendizaje de competencias es siempre funcional al discente (Tejada y Navío, 2019): su vinculación con el contexto y la 
necesidad de la acción implica planteamientos metodológicos y organizativos que sean abiertos, múltiples, variados y flexibles (Moreno, 2012; Tejada y Ruiz, 2013). La evaluación de competencias permiten integrar y conjugar diferentes modos de recoger evidencias (Cano, 2008; García-Sanz, 2014; Jornet et al., 2011; Tejada y Ruiz, 2013,2016). Una evaluación de competencias, por lo tanto, debe considerarlas como una interacción y movilización de saberes (Moreno, 2012; Muñoz y Araya, 2017). La evaluación de competencias supone valorar la capacidad del estudiantado para dar respuesta a situaciones con las que se va a encontrar en el futuro (De Rossi y Restiglian, 2019; García-Sanz, 2014).

Desde el diseño de la formación bajo el modelo competencial, Tejada y Ruiz (2013, 2016) optan por jerarquizar cada fragmento de lo que constituye una competencia: unidades de competencia, de las que derivan los resultados de aprendizaje como referente formativo. Esta atomización permite concretar lo holístico de la competencia en ámbitos donde se dice qué evaluar y cómo lograr las competencias esperadas a lo largo de un conjunto de cursos o de disciplinas, idealmente moduladas alrededor de una situación-problema (Díaz-Barriga y Barroso, 2014). Existe un momento donde la evaluación sí debe ser integral: en las prácticas o los trabajos de fin de grado.

La consideración de todos estos aspectos para establecer los principios básicos de una evaluación de competencias, revela el creciente aumento de complejidad cuando se hace una transición a un modelo competencial.

\subsubsection{Estándares, evidencias y consideraciones}

La evaluación de competencias exige estándares para evaluar analíticamente su dinámica interaccional, junto con la recopilación de evidencias que demuestren el cumplimiento de lo exigido. Un estándar corresponde a: "la especificación - u operativización - de los niveles de calidad que deben mostrarse como dominio de una competencia" (Jornet et al., 2011, p. 126), y sirven como nexo entre un concepto cualitativo de calidad y un concepto cuantitativo de evidencia. Los estándares devienen de criterios de evaluación que son definidos a priori según un principio, y que se consideran para decir que el desempeño del estudiantado fue satisfactorio y funcional, no solamente formal o declarativo (Rodríguez et al., 2017); además, entrega orientaciones para interpretar los resultados de una evaluación (García, 2010; Manríquez, 2012).

Varias personas autoras (Manríquez, 2012; Tejada y Ruiz, 2016) citan a la pirámide de Miller (1990) como un modelo para evaluar competencias, al segmentarlas según el nivel de saber involucrado en etapas que deben escalarse para considerar una competencia como desarrollada. Estos siguen la progresión saber $>$ saber cómo -> demostrar cómo -> hacer -> hacer y enseñar, que es un quinto nivel agregado por Tejada y Ruiz. Los primeros bloques aluden al saber como conocimiento, y los dos bloques posteriores corresponden a evaluar el saber como comportamiento.

Evaluar y enseñar, según un modelo de competencias, implica entrar en la lógica de los estándares competenciales (Jornet et al., 2011) mediante la recolección de evidencias. Las evidencias son las pruebas claras de los conocimientos, habilidades, destrezas y actitudes que una persona posee y que determinan su competencia. De acuerdo con García (2010), y Tejada y Ruiz (2016), las evidencias pueden clasificarse en evidencia de conocimiento (conjunto de conocimientos, teorías, principios y habilidades cognitivas que permiten el desempeño eficaz) y evidencia de desempeño (descripciones sobre variables o condiciones del comportamiento que permiten inferir que el desempeño fue logrado). Esto obliga a determinar qué tipos de actividades permitirán reunir qué evidencias.

Para Seidel, Stürmer, Prenzel, Jahn y Schäfer (2017), la recogida de evidencia implica identificar situaciones de aula que son decisivas en una instrucción de práctica efectiva. Los criterios de evaluación deben poder discriminar sobre la adquisición o no de la competencia (García-Sanz, 2014; Jornet et al., 2011). La evaluación por competencias tampoco puede focalizarse exclusivamente en objetivos concretos que solo beneficien el desenvolvimiento laboral (Muñoz y Araya, 2017); por ejemplo, un trabajo escrito puede evaluar 
la competencia de habilidad para investigar y escribir; habilidad útil, pero que no es la única que el alumnado requiere desarrollar y demostrar (González y Wagenaar, 2008). Se debe considerar, ante todo, que se está formando una persona integrante de la educación superior, para quien las demandas de desarrollo cognitivo superan a lo laboral. Incluso se puede aprovechar de la lógica del proceso centrado en el estudiantado para construir colaborativamente los criterios relevantes para valorar la calidad de un trabajo, lo que permite la apropiación de los criterios construidos en diálogo (Cano, 2015).

Hay que considerar los tiempos reales que se cuentan para evaluar. Un número elevado de competencias dificulta su adquisición y desarrollo real en el estudiantado y su evaluación (Villa y Poblete, 2011). Lo que se recomienda es enfocarse en un número que permita que su evaluación auténtica sea factible. Como se requiere tiempo para conseguir un nivel de desarrollo satisfactorio, habrá que reducir los contenidos del currículo, eligiendo los más importantes (Moreno, 2012).

A la hora de formular competencias, se debe pensar en que no sean demasiado abstractas, ya que no conducirían a acciones concretas para la mejora; sin embargo, unas competencias muy poco abstractas pueden llevar un nivel de detalle demasiado alto y hacer poco viable su aplicación (Bergsmann, Schultes, Winter, Schober y Spiel, 2015).

\subsubsection{Pasos de la evaluación}

Una vez declarada la complejidad detrás de la evaluación de competencias, es necesario observar cómo traducirla en un diseño viable.

Para considerar un diseño viable, se sugieren las directrices entregadas por el marco europeo (European Association for Quality Assurance in Higher Education (ENQA, 2015)): se diseñan con el fin de medir la obtención de los resultados buscados en aprendizaje y otros objetivos del programa; se adecuan a su propósito, sea este de diagnóstico, formativo o acumulativo; son elaborados por personas que conozcan la función de las evaluaciones en el progreso del estudiante hacia la adquisición de los conocimientos y habilidades relacionadas con la calificación; y no descansan, siempre que ello sea posible, en el juicio de una única persona examinadora.

La literatura ofrece varias opciones para establecer la separación entre los pasos a seguir al entablar la evaluación por competencias. A continuación, se revisan algunos:

Tobón (2013), divide los momentos de la evaluación de las competencias en:

1. Indagación: recoger información de forma sistemática en torno a cómo se están desarrollando las competencias, esto por medio de diversas estrategias y en consideración de sus componentes.

2. Análisis: la información obtenida en el paso anterior se analiza con base en los criterios y las evidencias requeridas, para así sacar conclusiones.

3. Decisión: a partir del paso anterior, se toman decisiones, las cuales pueden ser respecto al aprendizaje, las estrategias didácticas o la certificación.

4. Retroalimentación: compartir los resultados de la valoración, así: autovaloración, con docentes, compañeros y compañeras; covaloración, de pares con cada compañero y compañera; y heterovaloración, de la persona docente con cada estudiante. (p. 329)

Tejada y Ruiz (2013) proponen un modelo de concreción de los dispositivos de instrumentalización del con qué evaluar de 5 estrategias. Similar, pero expandido en 9 pasos, Jornet et al. (2011) proponen una estrategia metodológica para la planificación y el diseño de la evaluación de competencias:

1. Determinación y especificación del dominio competencial de la materia; qué va a ser evaluado.

2. Definición de criterios de evaluación. 
3. Especificación previa de un referente o estándar de ejecución, para determinar los criterios de evaluación.

4. Sistematización del proceso de recolección de evidencias.

5. Determinación del sistema de evaluación, instrumentos, recursos y técnicas de recogida de información: indicadores.

6. Definir un sistema de comparación de evidencias con el estándar.

7. Determinación del proceso de formación de la decisión según rangos establecidos.

8. Especificar los mecanismos de información a audiencias o retroalimentación para el estudiantado.

9. Diseñar los mecanismos o criterios de calidad del propio sistema de evaluación.

El modelo de Jornet es altamente centrado en la formulación de evidencias y criterios que resulten en el sistema de evaluación determinado. Además, la ruta que determina no aclara los espacios de participación. Bergsmann et al. (2015) prefieren ver la evaluación de competencias como un proceso sistemático de tres etapas, con un enfoque que se inspira más en los modelos de evaluación institucional como el de Stake (2006):

1. Evaluación del modelo de competencia teórica: se sugiere un focus group donde los grupos de interés discuten los criterios de calidad esperados del modelo de competencias.

2. Evaluación paralela del proceso de enseñanza y de las competencias reales del estudiantado a través de un sondeo a los grupos de interés: este es el momento ideal para encontrar brechas entre la teoría de competencias y la práctica. Se examina por medio de dos objetivos: identificar si hay una brecha entre el nivel ideal de competencia del estudiantado y el nivel de competencia del proceso de instrucción; e identificar la brecha entre el nivel ideal de competencia del estudiantado y su nivel de competencia real. En el sondeo no se espera tanta objetividad, y puede hacerse por autorreportes o encuestas online.

3. Evaluación detallada de aspectos concretos del proceso de enseñanza; este es el momento ideal para encontrar las razones de las fortalezas del modelo (donde no haya brechas) y las debilidades (donde sí las haya). Se le llama concreta porque se deben abordar objetivamente espacios donde surjan las brechas: en el currículo, en los métodos y formatos de enseñanza y examinación, en el proceso de aprendizaje del estudiantado, y en el contexto.

Bergsmann et al. (2015) se centran en varios componentes de enseñanza relevantes para el aprendizaje del estudiantado: (1) claridad de objetivos, (2) apoyo de las y los maestros, y (3) clima de aprendizaje. En esta estrategia se toma distancia de otros modelos al dividir la responsabilidad en los grupos de interés, incluido el estudiantado. La evaluación de competencias reales, además, permite poner en práctica un modelo realmente centrado en el estudiantado. En contraste, el rol del personal docente es minimizado en comparación con otras estrategias.

En síntesis, existen variadas opciones de pasos posibles para establecer una evaluación por competencias. Incluso, en un contexto constructivista, es visible que hay unas estrategias que son más centradas en la persona docente y otras más centradas en el estudiantado, razón por la que una estrategia equivocada puede resultar en la ofuscación de ciertos grupos de interés. Lo importante es tener conciencia de que se debe planificar de antemano, establecer estrategias para construirla, y realizarlo de manera metódica.

\subsection{Agentes de la evaluación de competencias}

Dadas las características de la competencia, no existe un agente evaluador único. La complejidad de las competencias implica procesos de autoevaluación, heteroevaluación y coevaluación (García-Sanz, 2014; Tejada y Ruiz, 2016; Tobón, 2013). Manríquez (2012) concibe la evaluación de competencias como una 
intersección entre docente, discente y contenidos. Hay que advertir que no todas las personas protagonistas tienen el mismo peso e incidencia en el proceso de evaluar (Muñoz y Araya, 2017).

Tobón (2013) prefiere el elemento socioformativo de los tipos de evaluación, usando el concepto de valoración para fines de evaluar competencias. La valoración de las competencias requiere de la interdependencia de los cuatro procesos diferenciados por el agente que los ejecuta: heterovaloración, autovaloración, covaloración y metavaloración.

Se deben establecer criterios para poder visualizar y evidenciar los logros obtenidos por el alumnado, docentes e inclusive por la misma institución de educación superior (Muñoz y Araya, 2017; Tejada y Navío, 2019). A continuación, se explorará en estos agentes:

\subsubsection{El rol del estudiantado}

Una evaluación centrada en el estudiantado significa que este grupo tiene el poder de analizar de forma activa su propio aprendizaje por medio de la autorregulación, y al usar como base criterios concretos sobre niveles de desarrollo, en un entorno de ayuda en el que la retroalimentación es inmediata, frecuente y formativa (Villa y Poblete, 2011). Se define autorregulación como "el control que ejercen los estudiantes sobre su cognición, comportamiento, emociones y motivación mediante el uso de estrategias para conseguir sus metas de aprendizaje" (Gómez y Romero, 2019, p. 96).

La evaluación de competencias debe contribuir a estos procesos de autorregulación. Los instrumentos y diseños no solo deben responder al desarrollo de una tarea específica, también tienen que implicar niveles de pensamiento y desafíos que incluyan situaciones o contextos similares al de la realidad (Muñoz y Araya 2017). La mera reproducción de la respuesta es un fallo de la evaluación por competencias; el objetivo de la evaluación debe ser la construcción de esta respuesta (García-Sanz, 2014).

La complejidad añadida de la evaluación de competencias permite distinguir entre mediciones de aprendizaje y mediciones del nivel de competencia (Caspersen, Smeby y Olaf Aamodt, 2017). El alumnado que empieza desde un nivel bajo de conocimiento puede experimentar un acelerado nivel de crecimiento y destacarse en el cumplimiento de la competencia, sin llegar a un nivel de conocimiento que pueda considerarse alto. Inversamente, una persona estudiante con un alto nivel de conocimiento puede experimentar un reducido crecimiento y cumplir solo parcialmente en el logro de la competencia, pero mantener un alto nivel de conocimiento a la hora de graduarse; el crecimiento de este alumnado depende de los procesos de autorregulación.

Para Villa y Poblete (2011), la autorregulación puede constituir una buena base para el aprendizaje orientado al logro de competencias. El estudio de van Dinther, Dochy, Segers y Braeken (2014) en estudiantes de educación superior demuestra que hay una alta correlación entre aprobación de competencias y autoeficacia (uno de los componentes de la autorregulación, son autopercepciones que tienen las personas sobre lo que logran con sus capacidades), y que la eficacia del estudiantado puede predecir el logro de las competencias en evaluaciones de final de año. Gómez y Romero (2019) observan una relación similar entre autorregulación y rendimiento académico.

Rodríguez-Gómez e Ibarra-Sáiz (2015) distinguen tres desafíos en la evaluación desde la perspectiva del estudiantado: (a) participación en la evaluación de su propio aprendizaje; (b) proalimentación (feedforward), que se centra en la entrega de información sobre los resultados de la evaluación a utilizarse de manera proactiva; $\mathrm{y}(\mathrm{c})$ producir tareas de evaluación de alta calidad.

Es poco lo que puede aportar la enseñanza por competencias y su evaluación si no se siguen los designios de Kemmis (1988) de una evaluación democrática, participativa y liberadora. El uso de la competencia en particular permite hacer distinción entre estados del progreso del estudiantado; si no se considera la autonomía y las necesidades del estudiantado, y si tampoco se le integra al proceso, se repetirán los modelos jerarquizados y horizontalizados de evaluar. 


\subsubsection{El rol del personal docente}

El enfoque en competencias pone el protagonismo del proceso de aprendizaje en la persona estudiante. El personal docente es solo un guía que media el aprendizaje, por lo que su instrucción debe conducir a estrategias (Tamez y Leyva, 2018) y condiciones (Le Boterf, 2000) que le permitan facilitar el conocimiento, y colaborar y guiar los niveles de aprendizaje (Muñoz y Araya, 2017). Tobón (2013) comparte la idea de que el éxito de los procesos de evaluación de las competencias está relacionado con el grado en el cual estos sean asumidos como válidos por el estudiantado, lo que es solo posible si se crean espacios para discutir sobre la importancia de la evaluación, sus tipos y estrategias.

Mas no se debe descuidar el rol docente como agente evaluador. En Cano (2008), la presencia de autorregulación conlleva a que el personal docente tenga dos tareas importantes: articular mecanismos de retroalimentación que ayuden al estudiantado a aprender, y establecer mecanismos y estrategias que ayuden al alumnado en el proceso de tomar conciencia de qué aprende y cómo lo hace.

Algunas personas autoras (Barrón-Tirado, 2009; Moreno, 2012) ponen el énfasis de la transición a las competencias en el cuerpo docente, ya que significa un enorme reto para dicho grupo. La enseñanza y la evaluación de competencias exigen profesionalismo, capacidad de innovación y compromiso con un cambio genuino en las concepciones y prácticas de evaluación del aprendizaje. Hay elementos que en la evaluación tradicional no se cuestionan: el por qué evaluar, y el qué hacer con los resultados de la evaluación. Estos elementos demandan del personal docente una postura crítica respecto a su tarea evaluadora (Cordero, 2018; Moreno, 2012).

Consecuentemente, las nuevas demandas que produce una evaluación de competencias, terminan inhibiendo su aplicación entre el personal docente por distintas razones: no se siente, no tiene formación; falta de tiempo, motivación o sensibilización (Ion y Cano, 2012); o bien, ven en la competencia un carácter excesivamente formalista que asocian al enfoque tecnológico-tecnócrata de enseñanza, o se muestran reticentes a vincular instrumentos de evaluación a competencias concretas por la estrecha relación entre competencia y contexto (López, Benedito y León, 2016) u otras aprehensiones de corte ideológico (Ecclestone, 1999). La variedad y profundidad de instrumentos que demanda la evaluación de competencias debe ser también considerada.

\subsubsection{El rol de las instituciones}

El involucramiento de la institución es también importante en el proceso (Bergsmann et al., 2015). Villa y Poblete (2011) destacan la importancia de las relaciones entre estudiantes, docentes y empleadores para la evaluación de alta calidad. Las instituciones de educación superior están cambiando su enfoque de la educación centrada en el personal docente a la educación centrada en el alumnado, y están cambiando de currículos basados en contenido, a currículos basados en competencias, a través de procesos que requieren de sistemas de seguimiento y evaluación (Bezanilla, García-Olalla, Paños-Castro y Poblete-Ruiz, 2019; Tejada y Navío, 2019). De acuerdo con esta perspectiva, conducir una evaluación basada en competencias es también una intervención en el proceso de desarrollo organizacional.

A través del proceso de implementación, la institución no solo desarrolla la capacidad de evaluación, sino que también fomenta la discusión y, por lo tanto, mejora la comprensión común de las competencias estudiantiles. A raíz de su estudio, van Dinther et al. (2014) recomiendan que las instituciones de educación superior deberían prestar atención al monitoreo y mejora de la autoeficacia del estudiantado por su rol de predictor de sus logros futuros. El uso de competencias, y su posterior evaluación, benefician a las instituciones de educación superior al contar con estándares representados por instrumentos de medición y 
evaluación, que entregan información sobre qué se persigue y qué se logra en el estudiantado (Jornet et al., 2011).

En la adopción de una enseñanza por competencias, los informes internacionales destacan el rol de las instituciones para facilitar estos procesos. Documentos como el Standards and Guidelines for Quality Assurance in the European Higher Education Area de la European Association for Quality Assurance in Higher Education (ENQA), (2015) funcionan como directrices a nivel europeo para asegurar la calidad interna y externa de los programas universitarios, con estándares como:

Las instituciones deben asegurarse de que los programas se entreguen de una manera que aliente a los estudiantes a tomar un rol activo en la creación del proceso de aprendizaje, y que la evaluación de los estudiantes refleje este enfoque. (p. 12).

$\mathrm{O}$ "Las instituciones deben publicar información sobre sus actividades, incluidos los programas, que sea clara, precisa, objetiva, actualizada y de fácil acceso” (p. 15).

El rol de las instituciones en esto también requiere ciertos compromisos. Un obstáculo que declaran las personas docentes es estar esperando algún tipo de política institucional sobre qué hacer y cómo (Ion y Cano, 2012). Una mirada crítica al rol de la institución es que el personal docente no siempre ha tenido la oportunidad de estar en diálogo con sus superiores por la falta de una cultura colaborativa y de coordinación entre el personal universitario (Ion y Cano, 2012; López et al., 2016). Si la institución no adapta el proceso a instancias más colaborativas, se corre el riesgo de alienar al cuerpo docente del concepto de competencias y de su evaluación.

\section{Conclusiones y RECOMENDACIONES}

Este artículo tiene por intención servir de introducción a una discusión fundamentada para docentes, curriculistas o cualquier otra persona profesional involucrada en procesos de mejora e innovación curricular. Cada apartado presentado podría ser un punto de partida para una búsqueda extensiva de literatura. Sin embargo, es suficiente con que la persona lectora adquiera conciencia de todos los elementos a considerar en la evaluación de competencias, y cómo difiere de mantener las mismas tácticas de evaluación cuando se pasa a un modelo centrado en el estudiantado. La literatura demuestra que a menudo las personas docentes dicen que se han adaptado al uso de competencias, pero difícilmente se refleja en sus prácticas evaluativas (Cordero, 2018; Díaz-Barriga y Barroso, 2014; Tamez y Leyva, 2018).

De los conceptos de la evaluación de competencias, se puede concluir que estos se alinean con los conceptos de evaluación formativa y evaluación auténtica en su carácter constructivista. En lugar de asumir que las competencias simplifican la interacción docente-aprendiz a un intercambio, se recomienda aprovechar la potencia que tienen para construir saberes y haceres desde el aprendizaje comprehensivo y situado.

En cuanto a los principios necesarios para facilitar una evaluación por competencias, se espera entregar indicaciones para guiar el proceso de integración de una evaluación de las competencias que sea rigurosa y fundamentada. Es necesario determinar que la evaluación de competencias es un proceso, y que por tanto depende de rutas que deben predefinirse. Igualmente, las competencias dependen de los estándares y de la comparación de evidencias con el estándar. Aun siendo constructivistas, exigen que su aplicación tenga contrapartes en el mundo real, y no todos los modelos dan el mismo protagonismo a todas las partes por igual, por lo que esta debe ser una elección fundamentada y prudente.

Si bien estas personas autoras enfocan la problemática en la persona docente, otras reparan en el rol de las instituciones de educación superior. Las investigaciones revelan que el personal docente, además de reparos intrínsecos que pueda tener hacia el cambio, a menudo siente que las instituciones de educación superior le insta a formarse en competencias sin un andamiaje adecuado que le permita entender qué se espera de su actuar. La instalación de un modelo de competencias sin cuidado en los agentes puede acabar demasiado centrado en el diseño y muy poco en el paso a la práctica (Beneitone y Yarosh, 2015), o bien la 
instrucción se limita a formación instrumental, sin establecer las bases teóricas de metodologías y recursos, ni de componentes contextuales o reflexivos (Ion y Cano, 2012). También están las implicaciones de un aprendizaje centrado en el estudiantado, que se exploraron brevemente en este artículo. La evaluación no se limita a los paradigmas y a las estrategias que puedan existir, sino que también requieren de un diálogo entre las partes involucradas. La persona profesional involucrada en este proceso debe reconocer que se necesita la participación de estudiantes, docentes e instituciones.

Introducir en una institución de educación superior las competencias implica entender que existe un cuerpo teórico detrás de ellas con diversas posturas. La discusión hecha en torno a la evaluación de competencias, muestra que es un campo fértil, con diversas perspectivas y no siempre en unísono. Reconocer la importancia de factores teóricos, metodológicos, colaborativos e instrumentales es parte de la complejidad que suma en la evaluación de competencias.

\section{ReferenCias BibliográficAS}

Barrón-Tirado, M. C. (2009). Docencia universitaria y competencias didácticas. Perfiles Educativos, 31(125), (pp. $76-$ 87).

Beneitone, P. y Yarosh, M. (2015). Tuning impact in Latin America: is there implementation beyond design? Tuning Journal for Higher Education, 3(1), (pp. 187-216). doi: https://doi.org/10.18543/tjhe-3(1)-2015pp187-216

Bergsmann, E., Schultes, M. T., Winter, P., Schober, B. y Spiel, C. (2015). Evaluation of competence-based teaching in higher education: From theory to practice. Evaluation and Program Planning, 52, (pp. 1-9). doi: https://d oi.org/10.1016/j.evalprogplan.2015.03.001

Bezanilla, M. J., García-Olalla, A. M., Paños-Castro, J., y Poblete-Ruiz, M. (2019). A model for the evaluation of competence-based learning implementation in higher education institutions: Criteria and indicators. Tuning Journal for Higher Education, 6(2), (pp. 127-174). doi: https://doi.org/10.18543/tjhe-6(2)-2019pp127-174

Birtwistle, T., Brown, C. y Wagenaar, R. (2016). A long way to go ... A study on the implementation of the learningoutcomes based approach in the EU. Tuning Journal for Higher Education, 3(2), (pp. 429-463). doi: https://d oi.org/10.18543/tjhe-3(2)-2016pp429-463

Bruner, J. (1978). The role of dialogue in language acquisition. En A. Sinclair et al. (Eds.). The Child's Concept of Language (pp. 241-256). New York: Springer-Verlag.

Cano, E. (2008). La evaluación por competencias en la educación superior. Profesorado. Revista de Currículum y Formación de Profesorado, 12(3), (pp. 1-16).

Cano, E. (2015). Las rúbricas como instrumento de evaluación de competencias en Educación Superior: ¿ Uso o abuso? Profesorado. Revista de Curriculum y Formación de Profesorado, 19(2), (pp. 265-280).

Caspersen, J., Smeby, J. C. y Olaf Aamodt, P. (2017). Measuring learning outcomes. European Journal of Education, 52(1), (pp. 20-30). doi: https://doi.org/10.1111/ejed.12205

Centro Europeo para el Desarrollo de la Formación Profesional (CEDEFOP). (2008). Terminology of European education and training policy: a selection of 100 key terms. Luxemburgo: Office for Official Publications of the European Communities. Recuperado de https://www.cedefop.europa.eu/files/4064_en.pdf

Cordero, R. (2018). Las prácticas de evaluación del formador de profesores. Un estudio de caso de la escuela normal. En O. Leyva, F. Ganga, J. Tejada y A. Hernández (Eds.). La formación por competencias en la educación superior: alcance y limitaciones desde referentes de México, España y Chile (pp. 139-171). México: Tirant Humanidades.

Cubero-Ibáñez, J., Ibarra-Sáiz, M. S. y Rodríguez-Gómez, G. (2018). Propuesta metodológica de evaluación para evaluar competencias a través de tareas complejas en entornos virtuales de aprendizaje. Revista de Investigación Educativa, 36(1), (pp. 159-184). doi: https://doi.org/10.6018/rie.36.1.278301

De Rossi, M. y Restiglian, E. (2019). Hybrid solutions for didactics in higher education: An interdisciplinary workshop of 'Visual Storytelling' to develop documentation competences. Tuning Journal for Higher Education, 6(2), (pp. 175-203). doi: https://doi.org/10.18543/tjhe-6(2)-2019pp175-203 
Dewey, J. (1989). Cómo pensamos. Nueva exposición de la relación entre pensamiento reflexivo y proceso educativo. Barcelona: Paidós.

Díaz-Barriga, F. y Barroso, R. (2014). Diseño y validación de una propuesta de evaluación auténtica de competencias en un programa de formación de docentes de educación básica en México. Perspectiva Educacional, 53(1), (pp. 36-56). doi: https://doi.org/10.4151/07189729-Vol.53-Iss.1-Art.210

Ecclestone, K. (1999). Empowerng or ensnaring?: The implications of outcome-based assessment in higher education. Higher Education Quarterly, 53(1), (pp. 29-48). doi: https://doi.org/10.1111/1468-2273.00111

European Association for Quality Assurance in Higher Education (ENQA). (2015). Standards and Guidelines for Quality Assurance in the European Higher Education Area (ESG). Bruselas: European Association of Institutions in Higher Education. Recuperado de https://www.enqa.eu/wp-content/uploads/2015/11/ESG_2015.pdf

García, B. (2010). Modelos teóricos e indicadores de evaluación educativa. Revista Electrónica Sinéctica, 35, (pp. 1-21).

García-Sanz, M. P. (2014). La evaluación de competencias en Educación Superior mediante rúbricas: un caso práctico. Revista Electrónica Interuniversitaria de Formación del Profesorado, 17(1). doi: https://doi.org/10.6018/reifop .17.1.198861

Gómez, J. y Romero, A. (2019). Enfoques de aprendizaje, autorregulación y autoeficacia y su influencia en el rendimiento académico en estudiantes universitarios de Psicología. European Journal of Investigation in Health, Psychology and Education, 9(2), (pp. 95-107). doi: http://doi.org/10.30552/ejihpe.v9i2.323

González, J. y Wagenaar, R. (2008). La contribución de las Universidades al proceso de Bolonia. Deusto: Universidad Groningen.

Ion, G. y Cano, E. (2012). La formación del profesorado universitario para la implementación de la evaluación por competencias. Educación XX1: Revista de La Facultad de Educación, 15(2), (pp. 249-270).

Isaacs, A. K. (2017). Tuning and History: A personal overview. Arts and Humanities in Higher Education, 16(4), (pp. 403-409). doi: https://doi.org/10.1177/1474022216686506

Jornet, J. M., González, J., Suárez, J. M. y Peraless, M. J. (2011). Diseño de procesos de evaluación de competencias: Consideraciones acerca de los estándares en el dominio de las competencias. Bordon, 63(1), (pp. 125-145).

Kemmis, S. (1988). El curriculum: más allá de la teoría de la reproducción. Madrid: Morata.

Le Boterf, G. (2000). Ingeniería de las competencias. Barcelona: Gestión 2000/EPISE.

López, C., Benedito, V. y León, M. J. (2016). El enfoque de competencias en la formación universitaria y su impacto en la evaluación. La perspectiva de un grupo de profesionales expertos en pedagogía. Formación Universitaria, 9(4), (pp. 11-22). doi: https://doi.org/10.4067/S0718-50062016000400003

Manríquez, L. (2012). ¿Evaluación en competencias? Estudios Pedagógicos, 38(1), (pp. 353-366). Recuperado de htt ps://doi.org/10.4067/S0718-07052012000100022

Miller, G. E. (1990). The assessment of clinical skills/competence/performance. Academic Medicine, 65(9), (pp. S63S67). doi: https://doi.org/10.1097/00001888-199009000-00045

Moreno, T. (2012). La evaluación de competencias en educación. Sinéctica, 39, (pp. 1-20).

Muñoz, D. R. y Araya, D. H. (2017). The challenges of competence-based assessment in the educational field. Educação e Pesquisa, 43(4), (pp. 1073-1086). doi: http://doi.org/10.1590/S1678-4634201706164230

Murga-Menoyo, M. Á. (2015). Competencias para el desarrollo sostenible: las capacidades, actitudes y valores meta de la educación en el marco de la Agenda global post-2015. Foro de Educación, 13(19), (pp. 55-83). doi: https ://doi.org/10.14516/fde.2015.013.019.004

Nodine, T. R. (2016). How did we get here? A brief history of competency-based higher education in the United States. The Journal of Competency-Based Education, 1(1), (pp. 5-11). doi: http://doi.org/10.1002/cbe2.1004

Pérez, M. (2014). Evaluación de competencias mediante portafolios. Perspectiva Educacional, 53(1), (pp. 19-35). doi: https://doi.org/10.4151/07189729-Vol.53-Iss.1-Art.213

Perrenoud, P. (2004). Diez nuevas competencias para enseñar. Barcelona: Graó. 
Rodríguez-Gómez, G. y Ibarra-Sáiz, M. S. (2015). Assessment as Learning and Empowerment: Towards Sustainable Learning in Higher Education. En M. Peris-Ortiz y J. M. M. Lindahl (Eds.), Sustainable Learning in Higher Education. Developing Competencies for the Global Marketplace (pp. 1-20). Cham, Suiza: Springer.

Rodríguez, M., Rodríguez, C. y Silva, L. H. (2017). Evaluación de competencias de investigación mediante reportes experimentales: un ejemplo con profesores de bachillerato. Interacciones. Revista de Avances En Psicología, 3(3), (pp. 111-119). doi: https://doi.org/10.24016/2017.v3n3.69

Sadler, D. R. (1989). Formative assessment and the design of instructional systems. Instructional Science, 18(2), (pp. 119-144). doi: https://doi.org/10.1007/BF00117714

Seidel, T., Stürmer, K., Prenzel, M., Jahn, G. y Schäfer, S. (2017). Investigating Pre-service Teachers' Professional Vision Within University-Based Teacher Education. En D. Leutner, J. Fleischer, J. Grünkorn y E. Klieme (Eds.), Competence Assessment in Education. Methodology of Educational Measurement and Assessment (pp. 93-109). Cham, Suiza: Springer.

Stake, R. E. (2006). Evaluación comprensiva y evaluación basada en estándares. Barcelona: Graó.

Tamez, G. y Leyva, O. (2018). Análisis de los roles, métodos de enseñanza y formas de evaluación desde el punto de vista del profesor en el proceso de enseñanza-aprendizaje de competencias: Estudio de Caso en la Universidad Autónoma de Nuevo León, México. En O. Leyva, F. Ganga, J. Tejada y A. Hernández (Eds.), La formación por competencias en la educación superior: alcances y limitaciones desde referentes de México, España y Chile (pp. 121138). México: Tirant Humanidades.

Tardif, J. (2008). Desarrollo de un programa por competencias: de la intención a su implementación. Profesorado. Revista de Currículum y Formación de Profesorado, 12(3), (pp. 1-16). Recuperado de: http://www.ugr.es/local /recfpro/rev123ART2.pdf

Tejada, J. (1999). Acerca de las competencias profesionales. Revista Herramientas, 56, (pp. 20-30).

Tejada, J. y Navío, A. (2019). Valoración de la Adquisición de Competencias Profesionales en el Prácticum a través del Contrato de Aprendizaje por parte de los Alumnos: Caso del Grado de Pedagogía. Revista Iberoamericana de Evaluación Educativa, 12(2), (pp. 67-87). doi: https://doi.org/10.15366/riee2019.12.2.004

Tejada, J. y Ruiz, C. (2013). Significación del prácticum en la adquisición de competencias profesionales que permiten la transferencia de conocimiento a ámbitos propios de la acción docente. Profesorado. Revista de Currículum y Formación del Profesorado, 17(3), (pp.91-110).

Tejada, J. y Ruiz, C. (2016). Evaluación de competencias profesionales en educación superior: Retos e implicaciones. Educación XX1, 19(1), (pp. 17-38). doi: https://doi.org/10.5944/educXX1.12175

Tobón, S. (2013). Formación integral y competencias. Pensamiento complejo, curriculo, didáctica y evaluación (4ta. Ed.). Colombia: Ecoe Ediciones.

van Dinther, M., Dochy, F., Segers, M. y Braeken, J. (2014). Student perceptions of assessment and student self-efficacy in competence-based education. Educational Studies, 40(3), (pp. 330-351). doi: https://doi.org/10.1080/030 55698.2014 .898577

Vygotsky, L. (1988). El desarrollo de los procesos psicológicos superiores. México: Crítica Grijalbo.

Villa, A. y Poblete, M. (2011). Evaluación de competencias genéricas: oportunidades y limitaciones. Bordón. Revista de Pedagogía, 63(1), (pp. 147-170).

Zlatkin-Troitschanskaia, O., Shavelson, R. J. y Kuhn, C. (2015). The international state of research on measurement of competency in higher education. Studies in Higher Education, 40(3), (pp. 393-411). doi: https://doi.org/1 $0.1080 / 03075079.2015 .1004241$

\section{Notas}

[1] http://tuningacademy.org/

\section{INFORMACIÓN ADICIONAL}


Revista Educación, 2021, vol. 45, NúM. 2, Julio-Diciembre, ISSN: 0379-7082 2215-2644.

Cómo citar: Bonnefoy Valdés, N. (2021). Evaluación de competencias en educación superior: conceptos, principios y agentes. Revista Educación, 45(2). Recuperado de http://doi.org/10.15517/revedu.v45i1.43444 